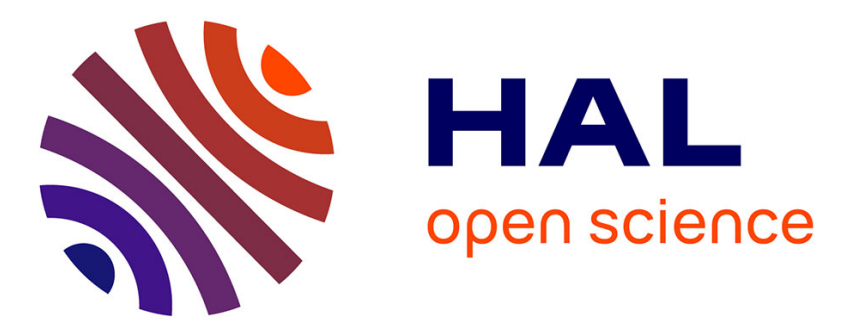

\title{
5G-microwave Tracking Performance Characterization
} Auryn Pink Soderini, Paul Thevenon, Christophe Macabiau, Laurent Borgagni, John Fischer

\section{To cite this version:}

Auryn Pink Soderini, Paul Thevenon, Christophe Macabiau, Laurent Borgagni, John Fischer. 5Gmicrowave Tracking Performance Characterization. ION GNSS+ 2019, 32nd International Technical Meeting of the Satellite Division of The Institute of Navigation, Sep 2019, Miami, United States. pp.2285-2298, 10.33012/2019.17016 . hal-02555023

\section{HAL Id: hal-02555023 \\ https://hal-enac.archives-ouvertes.fr/hal-02555023}

Submitted on 9 Jun 2020

HAL is a multi-disciplinary open access archive for the deposit and dissemination of scientific research documents, whether they are published or not. The documents may come from teaching and research institutions in France or abroad, or from public or private research centers.
L'archive ouverte pluridisciplinaire HAL, est destinée au dépôt et à la diffusion de documents scientifiques de niveau recherche, publiés ou non, émanant des établissements d'enseignement et de recherche français ou étrangers, des laboratoires publics ou privés. 


\title{
5G-microwave Tracking Performance Characterization
}

\author{
Auryn P. Soderini, Paul Thevenon, Christophe Macabiau, Ecole Nationale de l'Aviation Civile \\ Laurent Borgagni, John Fischer, Orolia
}

\section{BIOGRAPHIES}

Auryn Soderini is a Ph.D. student in the Department of Science and Air Navigation Engineering at the French National School of Civil Aviation, Toulouse, France. He received his M.Sc. degrees from Tampere University of Technology, Finland, in 2016. His current research interests focus on signal processing for positioning and navigation in mobile networks.

Dr. Paul Thevenon obtained a Ph.D. in the signal processing laboratory of ENAC. From 2010 to 2013, he was employed by CNES to supervise GNSS research activities. Since 2013, he is employed by ENAC as an Assistant Professor. His current activities are GNSS signal processing, integrity monitoring and hybridization.

Christophe Macabiau graduated as an electronic engineer in 1992 from the ENAC (Ecole National de l'Aviation Civile) in Toulouse, France. He received his Ph.D. in 1997. Since 1994 he has been working on the application of satellite navigation techniques to civil aviation and has been in charge of the TELECOM Lab of the ENAC since 2000.

Laurent Borgagni is a Research and Development Director and John Fischer is an Advanced Research and Development Vice President at Orolia, a world leader in GPS-based time and frequency equipment.

\begin{abstract}
This paper evaluates the theoretical performance of signal tracking in a noisy single-path environment using 5G-microwave and LTE signals. Such OFDM signals suit navigation applications thanks to build-in symbol sequences which despite being designed for communication services since are known at the receiver can be exploited for pseudorange computation. By using multiple numerologies, $5 \mathrm{G}$ is putting into place an important degree of flexibility which was not explored before. It turns out, the numerology counts towards the signal tracking performance. However, the nominal tracking capacity expressed by 5Gmicrowave signals at the highest sampling frequency is reduced due to the lack of full bandwidth useful signals. Top-ranked LTE-CRS performance can hypothetically improve by aggregating multiple carrier components.
\end{abstract}

\section{INTRODUCTION}

Today, the world is crowded with communication devices and radio signals which, despite not being designed for navigation, still carry a certain degree of information for location purposes. The propagation environment and related system dynamics can be regarded as navigation reference sources. Such signals that rely on an existing infrastructure are notably appropriate to shape cost-effective navigation solutions.

Worldwide mobile wireless networks are most densely deployed in urban areas. Here, buildings, trees and other elements such as interference and spoofing can reduce the performance of navigation systems exclusively based on GNSS. Therefore, a resilient navigation solution based on current long terms evolution (LTE) signals and future fifth generation (5G) signals is targeted. A communication receiver with built-in functions such as time synchronization and channel estimation measuring received signal time delay can further be completed by additional functions to meet demanding positioning accuracy requirements. Extracting time delay information is seen as the natural way to address localization opportunistically, although other ways using different metrics such as angle of arrival, not primarily focused in this paper, may be pursued.

In the recent years, LTE signals have been regarded with interest for navigation. Some interesting features that make these signals attractive are the high bandwidth employed, which can be $20 \mathrm{MHz}$ at the most, and their high received power in urban situations. Among the signals that can be used for opportunity navigation, synchronization signals (xSSs), namely primary synchronization signals (PSSs) and secondary synchronization signals (SSSs), and cell-specific reference signals (CRSs) have been regarded notably. These signals, namely ranging signals (RSs), can be used to estimate the channel impulse response (CIR) by which time of arrival (TOA) can be extrapolated. The CIR estimate is obtained by correlating xSS (CRS), extracted from the time frame synchronized received signal, with the local replica ideally matching XSS (CRS). Once the CIR is estimated, the time of arrival estimate can be obtained. The higher bandwidth of CRS signals (i.e. complex symbols scattered 
over the whole occupied bandwidth) translates into a better accuracy of TOA estimate as compared to that can be achieved by the smaller bandwidth xSS signals (i.e., continuous complex symbols allocated in $1 \mathrm{MHz}$ of frequency resources). One such TOA estimate is rough, hence used to start the delay tracking. In this stage, known as delay-locked loop (DLL), 3 different correlator outputs, calculating the correlation function at 3 different instants, are combined into a discriminator to obtain the update value of the delay estimate. The noise variance of the discriminator function decreases as a function of the carrier to noise density ratio [1], [2], [3]. The impact of multipath on the ranging error decreases as a function of the signal bandwidth [1]. Hence, CRSs are top-ranked signals for opportunity navigation based on LTE.

Several papers discussed the structure of LTE signals and therein ranging signals, and characterized the tracking performance for such signals. However, to the best of our knowledge, there were rare publications addressing the tracking performance of near future $5 \mathrm{G}$-microwave signals.

The commercial deployment of 5G systems is planned for the year 2020 through microwave deployment e.g., frequencies in the sub- $6 \mathrm{GHz}$ spectrum. As $5 \mathrm{G}$ microwaves will be deployed in one year, the current number of undefined elements with respect to the system initial phase is expected to be very small. Consequently, the degree of system exploration for positioning is expected to be good enough. 5G sub-6 GHz signals have high central frequency ( $3.5 \mathrm{GHz}$ ) and large bandwidth (up to 100 $\mathrm{MHz}$ ) compared to LTE signals (e.g. carrier frequency $2.8 \mathrm{GHz}$, bandwidth $20 \mathrm{MHz}$ ), therefore they offer potentially increased tracking performance, while they can be associated with a signal coverage that is comparable with LTE.

This paper discusses the signal characteristics and evaluates the expected tracking performance for LTE and 5G sub- $6 \mathrm{GHz}$, comparatively

The general structure of the document is as follows. The first section of the paper is an introduction. The second section is a description of the acquisition and tracking of signals in orthogonal frequency division multiplexing (OFDM) systems to derive pseudorange measurements. The discriminator function model of the delay tracking loop and its error variance due to noise are discussed. The third section is a description of LTE and 5G-microwave signals. The signal architecture is described with focus on ranging signal components being compared and discussed. In the fourth section, the signal tracking performance is demonstrated for the two communication systems, and for the different ranging signals and system configurations. Finally, the conclusions are drawn, and future work is identified.

\section{OFDM SIGNAL TRACKING FOR PSEUDORANGE MEASUREMENT}

\subsection{OFDM signal structure}

Orthogonal frequency division multiplexing is a multicarrier transmission scheme that divides the system bandwidth into several narrow equally spaced sub-bands, referred to as subcarriers. These subcarriers are characterized by the following principal properties. Firstly, subcarriers are orthogonal sequences. It means that the spectral maximum of each subcarrier occurs at the zero-crossing frequency of the remaining subcarriers in the series. Therefore, each subcarrier spectrum can partially overlap to each other without causing interference. This permits to use the spectrum resources efficiently. However, under the non-ideal synchronization statement, synchronization errors reduce the orthogonality among subcarriers and produce intercarrier interference (ICI). Thus, spectrum efficiency comes at the expense of synchronization error sensitivity, which constrains the subcarrier spacing design relative to system requirements. Secondly, subcarriers are narrowband. As such, they individually experience a nearly flat channel transfer function even if the overall channel is frequency selective. More precisely, this condition is fulfilled when the spectrum amplitude variations of the channel are small enough compared to the subcarrier bandwidth. In this respect, channel equalization and subsequent symbol detection in the receiver become easy, which is a key feature for transmission occurring in propagation channels affected by heavy multipaths. Lastly, subcarriers have separate modulation schemes that can be adapted to the frequency channel variations in flexible manner, or even muted.

In multipath propagation environments, inter-symbol interference (ISI) might occur when the transmitted symbol duration is small compared to the maximum multipath delay. Such interference introduces channel distortion that degrades the subcarriers orthogonality due to the time spread of contiguous transmitted symbols. To cope with this phenomenon the duration of each transmitted symbol is artificially extended in the transmitter by a guard interval, also known as cyclic prefix (CP). Precisely, the cyclic prefix is a copy of the terminal portion of the symbol appended at the symbol front, which allows to increase the overall symbol duration with no need to reduce the subcarrier spacing, and which makes the symbol demodulation robust to symbol start synchronization error. This higher degree of freedom obviously comes at the expense of data rate.

OFDM signals are designed to accommodate data symbols, specifically complex symbols, onto a two-dimensional timefrequency physical layer. The digital waveform is nothing but a sequence of individually encoded blocks of data symbols, referred to as OFDM symbols. The encoding operation that maps individual blocks of data symbols to OFDM symbols is the inverse fast Fourier transform (IFFT). Likewise, the fast Fourier transform (FFT) decodes OFDM symbols to data symbol blocks. Note that the FFT is an efficient processing unit. Thus, it supports a relatively high number of input samples at once for which the processing computation is low enough for typical system requirements. The number of parallel samples at both 
side of the IFFT must be of the form $2^{N}$ with $N$ system parameter. This number that matches the samples within the symbol excluding the cyclic prefix, also matches the size of the FFT used for encoding and decoding the data symbols.

Note that the signal bandwidth is defined by the sampling frequency $F_{s}$ or inverse of sampling interval $T_{s}$. Thus, the sampling frequency divided by the total number of subcarriers returns the subcarriers spacing $\Delta_{c}$, which is the frequency resolution of subchannels. Likewise, the sampling interval multiplied by the total number of subcarriers returns the duration of the OFDM symbol $\Delta_{S}$ (without cyclic prefix). As subcarrier spacing meets the inverse of symbol duration, an increase in the total number of subcarriers under the same bandwidth translates into a longer symbol duration and, therefore, a more robust multipath transmission. The main OFDM parameters are summarized in Table 1.

\section{Baseband channel model}

The digital form of the channel impulse response can be written as

$$
h_{i, n}=\sum_{l=0}^{L_{i}-1} a_{i, l} \delta_{n-\tau_{i}-\tau_{i, l}^{\prime}}
$$

where $i$ is the symbol index, $n$ is the discrete time reference, $a_{i, l}$ is a complex scale factor, $\delta$ denotes the Dirac delta function and $L$ is the number of multipaths; $\tau_{i}$ and $\tau_{i, l}^{\prime}$ are respectively the delay of the direct component and the delay of the multipath $l$ relative to the direct component both normalized by the sampling interval $T_{s}$. Since the subcarriers are narrowband, the channel impulse response is assumed to be constant over the $i$-th transmitted symbol duration $\Delta_{s^{\prime}}$.

The FFT of (1), written as

$$
H_{i, k}=\sum_{l=0}^{L_{i}-1} a_{i, l} e^{-j 2 \pi\left(\tau_{i}+\tau_{i, l}^{\prime}\right) k / N_{C}}
$$

is referred channel frequency response.

Table 1. OFDM parameters. Abbreviation: OFDM, orthogonal frequency division multiplexing

\begin{tabular}{llll}
\hline Notation & Unit & Equivalence & Description \\
\hline$N_{d}$ & - & - & Number of data subcarriers per OFDM symbol \\
$N_{g}$ & - & - & Number of guard subcarriers per OFDM symbol \\
$N_{c}$ & - & $N_{c}=N_{d}+N_{g}$ & Total number of subcarriers per OFDM symbol \\
$\Delta_{p}$ & {$[\mathrm{~Hz}]$} & - & Pilot spacing \\
$N_{p}$ & - & - & Number of pilot subcarriers in the OFDM symbol \\
$T_{s}$ & {$[\mathrm{~s}]$} & - & Sampling interval \\
$F_{s}$ & {$[\mathrm{~Hz}]$} & $F_{c}=1 / T_{s}$ & Sampling frequency \\
$\Delta_{c}$ & {$[\mathrm{~Hz}]$} & $\Delta_{c}=F_{s} / N_{c}$ & Subcarrier spacing \\
$\Delta_{p}^{\prime}$ & - & $\Delta_{p}^{\prime}=\Delta_{p} / \Delta_{c}$ & Normalized pilot spacing \\
$B_{d}$ & {$[\mathrm{~Hz}]$} & $B_{d}=N_{d} \Delta_{c}$ & Transmission bandwidth \\
$B_{g}$ & {$[\mathrm{~Hz}]$} & $B_{g}=N_{g} \Delta_{c}$ & Protection bandwidth \\
$B_{c}$ & {$[\mathrm{~Hz}]$} & $B_{c}=F_{s}=N_{c} \Delta_{c}$ & Signal bandwidth \\
$L$ & - & - & Number of samples within the cyclic prefix \\
$N_{t}$ & - & $N_{c}+L$ & Number of samples per symbol including the cyclic prefix \\
$\Delta_{s}$ & {$[\mathrm{~s}]$} & $\Delta_{s}=N_{c} T_{s}=1 / \Delta_{c}$ & OFDM useful symbol duration \\
$\Delta_{s^{\prime}}$ & {$[\mathrm{s}]$} & $\Delta_{s^{\prime}}=\Delta_{s}+L T_{\mathrm{s}}$ & OFDM symbol duration \\
\hline
\end{tabular}

\section{Baseband demodulated data model}

The received data symbols at the FFT output after timing and frequency synchronizations, and cyclic prefix removal, can be written as 


$$
Y_{i, k}^{\gamma, \epsilon}=e^{j \theta_{0}} e^{j \frac{2 \pi \gamma i N_{t}}{N_{c}}} \sum_{q=-\infty}^{\infty} h_{i, u} \sum_{n=0}^{N_{c}-1} x_{i, n_{\epsilon}-q} e^{-j \frac{2 \pi n(k-\gamma)}{N_{c}}}
$$

Before FFT, Equation (3) is a circular convolution between the channel impulse response and each transmitted symbol, and it includes both time and frequency residual errors. The notation in (3) can be summarized as

$i$ is the symbol index

$k$ is the subcarrier index

$\theta_{0}$ is a random initial phase

$\gamma$ is the residual carrier frequency offset normalized by $\Delta_{c}$

$\epsilon$ is the residual time offset normalized by $T_{S}$

$N_{t}$ is the number of samples per symbol including the cyclic prefix

$N_{c}$ is the number of subcarrier per symbol

$h$ is the channel impulse response digital representation

$x$ is the encoded baseband data at the IFFT output

$n_{\epsilon}=n+\tau-\epsilon$ with $n$ the symbol sample index; $\tau$ is the normalized delay of the direct component at the time of synchronizing; $\epsilon=\tau-\hat{\tau} ; \hat{\tau}$ is the $\tau$ estimate

The equation above becomes much reduced under the assumption of timing and/or frequency perfectly synchronized, which holds if the residual errors $\gamma$ and/or $\epsilon$ are nulls.

\subsection{Correlation with an OFDM signal over the pilot subcarriers}

In OFDM, reference data symbols known as pilots are transmitted at specific times and frequencies. In this context, bearing the signal structure in mind, we will use the terms time and symbol as well as frequency and subcarrier interchangeably. Given a pilot frequency index space $\mathcal{P}$, the transmitted pilots are modelled as

$$
P_{i, k}=\left\{\begin{array}{l}
X_{i, k}, \text { if } k \in \mathcal{P}_{i} \\
0, \text { otherwise }
\end{array}\right.
$$

where $X_{i, k}$ are the transmitted data symbols and $\mathcal{P}_{i}$ is the subcarrier index set for the pilots in the $\mathrm{i}$-th symbol.

Usually, pilot subcarriers are spaced with regular interval across subcarriers, i.e., 1 subcarrier out of $\Delta_{p_{i}}$ is a pilot subcarrier. In this case, as in LTE and $5 \mathrm{G}$ signals, it can be written,

$$
\mathcal{P}_{i} \Leftrightarrow k=m \Delta_{p_{i}}+a_{i}, \text { for } m=1,2, \cdots, N_{p_{i}}
$$

where $\Delta_{p_{i}}$ and $N_{P_{i}}$ are the pilot spacing and the total number of pilots for the $i$-th symbol, and $N_{g}<a_{i} \leq N_{c}-N_{g}$ is the first pilot index for the $i$-th symbol; $N_{g}$ is the number of guard subcarriers, $N_{c}$ is the total number of subcarriers. Note that $N_{g}$ and $N_{c}$ are common to all symbols.

The inclusion of pilots within a block of decoded data symbols is illustrated with an example in Figure 1.

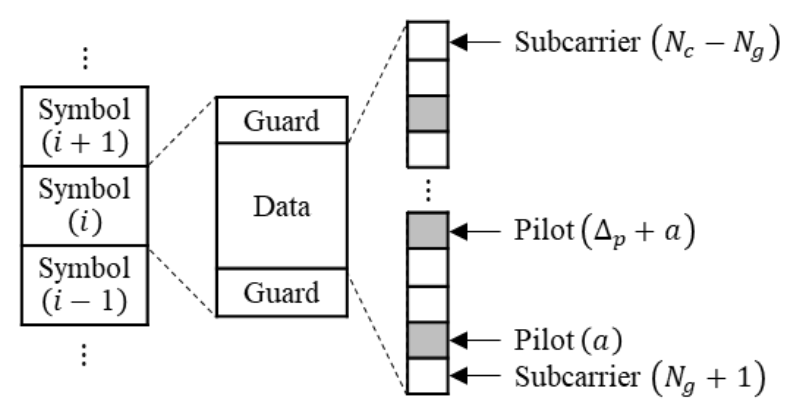

Figure 1. OFDM signal structure and pilot inclusion 
Let us assume $\gamma=0$ and $\epsilon=\tau$. Then, under the ideal statement of perfect frequency synchronization, the received data symbols at the receiver FFT output are the product between the transmitted data symbols at the transmitter IFFT input and the channel frequency response,

$$
Y_{i, k}^{\gamma=0, \epsilon=\tau}=X_{i, k} H_{i, k}
$$

Since the pilots are known, at the receiver, the channel impulse response can be estimated by correlating the received pilots and a local replica of the transmitted pilots as

$$
\hat{h}_{i, u}=\frac{1}{N_{p_{i}}} \sum_{k \in \mathcal{P}_{i}} Y_{i, k}^{\gamma=0, \epsilon=\tau} P_{i, k}^{*} e^{j \frac{2 \pi k u}{N_{c}}}
$$

which after replacing (2) in (6), and (6) in (7), becomes

$$
\hat{h}_{i, u}=\frac{\sigma_{p_{i}}^{2}}{N_{p_{i}}} \sum_{l=0}^{L_{i}-1} a_{i, l, u} \frac{\sin \pi \beta\left(\tau_{i}+\tau_{i, l}^{\prime}-u\right)}{\sin \pi \beta\left(\tau_{i}+\tau_{i, l}^{\prime}-u\right) / N_{p_{i}}}
$$

where $u$ is the normalized time shift of the local replica introduced by the correlation in (7), $\sigma_{p_{i}}^{2}$ is the power of the $i$-th pilot symbol, $a_{i, l, u}$ reflects the amplitude attenuation and the phase rotation of the $l$-th propagation component of the multipath channel relative to the $i$-th symbol, $\beta=\Delta_{p}^{\prime} N_{p} N_{c}^{-1}$.

It is shown in [2] that for a relatively small offset between the correlation delay and the delay from each received signal component (i.e. in (8), very small argument of the sine at the denominator), the channel impulse response can be approximated as

$$
\hat{h}_{i, u} \approx \sigma_{p_{i}}^{2} \sum_{l=0}^{L_{i}-1} a_{i, l, u} \operatorname{sinc} \pi \beta\left(\tau_{i}+\tau_{i, l}^{\prime}-u\right)
$$

where sinc $(\cdot)$ denotes the sinusoidal cardinalis function. Note that the CIR estimate is the convolution of the actual CIR by a sinc function.

\subsection{OFDM signal acquisition}

The OFDM reference symbols, which are enclosed in the transmitted signal and are known by the receiver can be used to obtain a symbol delay estimate $\hat{\tau}$ to find the symbol start and the carrier frequency offset (CFO) using a coarse synchronization algorithm [4].

After this coarse synchronization process the demodulated signal, if the carrier frequency offset (Doppler frequency, clock drift and transceiver oscillators mismatch) is perfectly compensated, can be expressed as:

$$
Y_{i, k}^{\gamma=0, \epsilon}=Y_{i, k}^{\gamma=0, \epsilon=\tau} e^{j \frac{2 \pi k \hat{\imath}}{N_{c}}}=X_{i, k} H_{i, k} e^{j \frac{2 \pi k \hat{\tau}}{N_{c}}}
$$

where $\epsilon=\tau-\hat{\tau}$ indicates the residual timing error.

Let us consider the propagation environment as an average white gaussian noise channel (AWGN). The channel adds a complex white noise, which is modelled as a zero-mean Gaussian random variable, to each data symbol. Let us denote with $\sigma^{2}$ the variance of the filtered AWGN. Let us define the cross-correlation function of the pilots in the $i$-th received symbol after synchronization $(\gamma=0)$ and after noise addition with their receiver local replica as

$$
\begin{aligned}
\hat{h}_{i, \epsilon}^{n} & =\hat{h}_{i, u+\hat{\tau}}+v_{i, \epsilon} \\
& =N_{p_{i}}^{-1} \sum_{k \in \mathcal{P}_{i}}\left(Y_{i, k}^{\gamma=0, \epsilon}+Z_{i, k}\right) P_{i, k}^{*} e^{j \frac{2 \pi u k}{N_{c}}}
\end{aligned}
$$


where $\hat{h}_{i, \epsilon}^{n}$ is the channel impulse response extrapolated by the $i$-th received OFDM symbol, after synchronization and after noise addition; where $v_{i, \epsilon}$ denotes the correlation of the local pilot replica of the transmitted pilots with the decoded channel noise $Z_{i, k} ; v_{i, \epsilon}$ and $Z_{i, k}$ are complex Gaussian noise with zero-mean and variance respectively $\sigma^{2} / N_{c}$ and $\sigma_{Z_{i, k}}^{2}=A \sigma^{2} /\left(N_{p_{i}} N_{c}\right)$ [2]; $A$ denotes $\mathrm{E}\left[\left|P_{i, k}^{2}\right|\right]$ with [·] statistical expectation.

After the CIR estimation, the matching pursuit (MP) algorithm [5] can be performed to obtain the delay estimates of each multipath component. Note that different algorithms performing acquisition delay estimate there exist in literature [6] [7]. The noise power in (11) can be reduced by doing the average over several symbols.

\subsection{OFDM signal tracking}

The estimation accuracy of the signal delay can be improved using a delay-locked loop which includes a discrimination function and a loop filter.

The discriminator function employs two locally generated pilot replicas, namely the early replica, $P_{i, k}^{E}$, and the late replica, $P_{i, k}^{L}$. The early replica is an anticipated copy of the original replica and the late replica is a delayed copy of the original replica. It can be written

$$
P_{i, k}^{E}=P_{i, k}^{L^{*}}=P_{i, k} e^{j \frac{\pi k \delta}{N_{c}}}
$$

where $(\cdot)^{*}$ denotes complex conjugate and $\delta$ is a design parameter known as correlator spacing which is defined as two times the absolute value of the correlator shift normalized by the sampling interval.

It can be shown that the cross-correlation function between the received signal and the early (or late) replica is a shifted copy of the cross-correlation (11), with a positive (or negative) shift that matches the one used for the replica generation. Thus, under the assumption of small correlation delay it can be written that

$$
\begin{aligned}
& \hat{h}_{i, \epsilon}^{E}=\hat{h}_{i, \epsilon-\delta / 2}^{n} \approx \sigma_{p_{i}}^{2} \sum_{l=0}^{L_{i}-1} a_{i, l, \epsilon-\delta / 2} \operatorname{sinc} \pi \beta(\epsilon-\delta / 2)+v_{i, \epsilon-\delta / 2} \\
& \hat{h}_{i, \epsilon}^{L}=\hat{h}_{i, \epsilon+\delta / 2}^{n} \approx \sigma_{p_{i}}^{2} \sum_{l=0}^{L_{i}-1} a_{i, l, \epsilon+\delta / 2} \operatorname{sinc} \pi \beta(\epsilon+\delta / 2)+v_{i, \epsilon+\delta / 2}
\end{aligned}
$$

Let us define $\tilde{h}_{i, \epsilon}^{E}=\left.\hat{h}_{i, \epsilon}^{E}\right|_{L_{i}=1}$ and $\tilde{h}_{i, \epsilon}^{L}=\left.\hat{h}_{i, \epsilon}^{L}\right|_{L_{i}=1}$. The propagation channel is assumed to have only the direct path to isolate the effect of the noise onto the delay error estimate from the effect of the reflected paths. Then, let us define the normalized early-minus-late-power (EMLP) discriminator as

$$
d_{i, \epsilon}^{\kappa}=\kappa_{i}^{-1} d_{i, \epsilon}=\kappa_{i}^{-1}\left(\left|\tilde{h}_{i, \epsilon}^{E}\right|^{2}-\left|\tilde{h}_{i, \epsilon}^{L}\right|^{2}\right)
$$

where the normalization factor $\kappa_{i}$ can be derived as

$$
\kappa_{i}=\left.\dot{s}_{i, \epsilon}\right|_{\epsilon \rightarrow 0}=\left.\frac{d s_{i, \epsilon}}{d \epsilon}\right|_{\epsilon \rightarrow 0}=\frac{16 A^{2}}{\pi^{2} \beta^{2} \delta^{3}}(\pi \beta(\delta / 2) \sin \pi \beta \delta+\cos \pi \beta \delta-1)
$$

where

$$
s_{i, \epsilon}=E\left[d_{i, \epsilon}\right]=A^{2}\left(\operatorname{sinc}^{2} \pi \beta(\epsilon-\delta / 2)-\operatorname{sinc}^{2} \pi \beta(\epsilon+\delta / 2)\right)
$$

is referred to as s-curve function.

In (16), $d a / d b$ denotes derivative of function $a$ with regards to variable $b$. In (17), $E[\cdot]$ denotes expected value. The normalization factor is the derivative of the s-curve in the linear region which is around the zero-crossing and it is used to make the slope of the discriminator function equal to one in the linear region. Therefore, the normalized discriminator output is equal to $\epsilon$ for small $\epsilon$; which means the discriminator estimate has no bias with respect to the actual tracking error.

The tracking loop filter can reduce the variance of the delay error at the discriminator output by a factor of $2 B_{l} T_{L}[8]$, with $B_{l}$ the one-side equivalent loop filter bandwidth expressed in Hertz and $T_{l}$ the loop update expressed in seconds. After filtering, 
the smoothed delay error estimate at the loop filter output, which denotes $\tilde{\epsilon}$, is used to update the time delay estimate of the next loop pilot sequence as $\hat{\tau}_{i+\Delta_{l}}=\hat{\tau}_{i}+\tilde{\epsilon}$, with $\Delta_{l}$ the loop update interval normalized by $\Delta_{s}$. Such delay estimates since translate into transmitter to receiver pseudo distance allow to perform a position computation. The position computation has not yet been developed and it is out of the scope of the paper.

If the total number of pilots is constant over the estimation symbols, the standard deviation of the delay error estimate at the loop filter output can be written using the result in [3]

$$
\sigma_{\tilde{\epsilon}}=\sqrt{2 B_{l} T_{l} \frac{4(1-\operatorname{sinc} \pi \beta \delta)(\operatorname{sinc} \pi \beta \delta / 2)^{2}}{c^{2} N_{p} \kappa^{2} \operatorname{SNR}}\left(1+\frac{(1+\operatorname{sinc} \pi \beta \delta)}{2 c^{2} N_{p}(\operatorname{sinc} \pi \beta \delta / 2)^{2} \mathrm{SNR}}\right)}
$$

where $\sigma_{\tilde{\epsilon}}$ is expressed in samples; SNR $=A^{2} / \sigma^{2}$ is the signal-to-noise ratio; $c$ is the ratio between the amplitude of a pilot subcarrier and the amplitude of a data subcarrier.

\section{LTE AND 5G SIGNALS DESCRIPTION FOR SIGNAL APPLIED STUDY}

Third Generation Partnership Project (3GPP) specifications contribute with the global scale standardization process, which is regulated by the global telecommunication standardization sector (ITU-T) of the International Telecommunication Union (ITU). The ITU International Mobile Telecommunication (IMT) system include evolving standard frameworks, such as IMTAdvanced and IMT-2020 for LTE and 5G industry, respectively.

\subsection{LTE signal structure}

The frame structure of the LTE signal is defined by the European Telecommunication Standards Institute (ETSI), a European standards organization (ESO) partner with 3GPP, in its technical specification document [9].

In frequency division duplex (FDD), the up and down communication links are separated in frequency. The signal structure for FDD is organized in frames, sub-frames and slots, where each frame $(10 \mathrm{~ms})$ is divided into 10 subframes and each subframe ( $1 \mathrm{~ms})$ is divided into 2 slots $(0.5 \mathrm{~ms})$. The slot structure is detailed in Figure 2.

The signal structure is designed to meet cellular radio network requirements such as capacity and coverage over different configurations. The channel bandwidth ranges from $1.4 \mathrm{MHz}$ to $20 \mathrm{MHz}$ through six possible choices, while the cycle prefix can have either a short or extend format. The extended CP is used for larger cell size as the amount of delay spread of the radio channel becomes more significant.

The minimum amount of transmission resource (in terms of number of subcarriers and transmit symbols) that can be allocated to the user terminal in a time slot is denoted resource block (RB). For normal CP, a resource block consists of 12 subcarriers interspaced with $\Delta_{c}=15 \mathrm{kHz}$, and 7 symbols. The first symbol $(5.21 \mu \mathrm{s})$ in the resource block is longer than the regular symbol duration $(4.69 \mu \mathrm{s})$. The total number of resource blocks in one slot increases as a function of the deployed channel bandwidth. The resource grid for one subframe is showed in Figure 3.

\section{Frequency bands}

The European Communication Office (ECO) reported information on the licensing of mobile frequency bands for the European Conference of Postal and Telecommunications Administrations (CEPT) member countries [10]. Network operators provide mobile service through standardized 3GPP LTE bands [11]. In Europe, LTE is deployed at 0.7, 0.8, 1.8, 2.1 and 2.6 GHz bands.

\section{Cell-specific reference signals}

Cell-specific reference signals are designed for channel estimation, which permits channel equalization and data demodulation under channel distortion conditions. These signals are generated by using pseudo-random generators. The signal generation depends on four parameters, namely the slot number within a radio frame, the OFDM symbol number within the slot, the cell identification code, and the type of cyclic prefix being either normal or extended [9]. The length of these signals which are

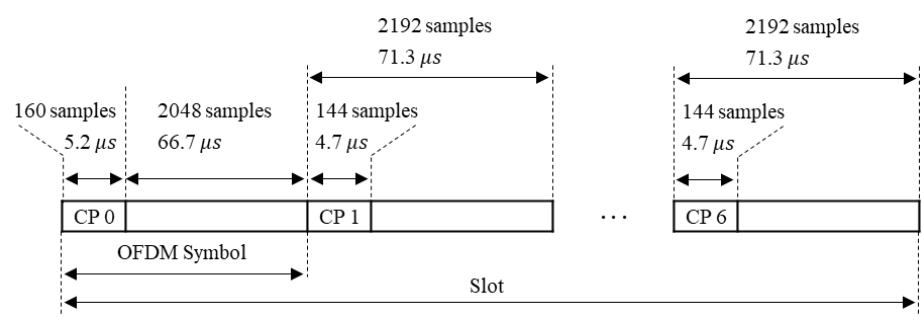

Figure 2. One slot structure for the $20 \mathrm{MHz}$ channel bandwidth use case 


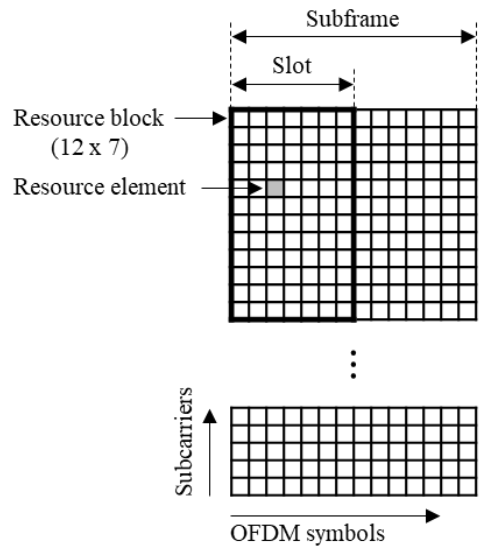

Figure 3. Downlink subframe resource grid. Adapted from primary source [9]

transmitted in every subframe and over the whole operating bandwidth is proportional to the maximum allowed number of resource blocks. It means that there are two elements of the sequence in each resource block at the given OFDM symbol. In case of multiple antennas, one reference signal can be mapped in different antenna ports. The way with which this is ruled is signal type wise. In general, the resource elements used for cell-specific reference signals on one antenna port are not used on any other antenna ports but set to zeros. The CRS signal structure is summarized in Table 2.

\section{Synchronization signals}

The standard [9] defines two sets of signals, the primary synchronization signals, PSSs, linked to the physical-layer cell identity, and the secondary synchronization signals, SSSs, linked to the physical-layer cell identity group. These signals are transmitted in the downlink to provide the receiver with four informative elements: time and frequency offset estimates; the beginning of received radio frames; the operated duplexing schemes; and the physical identity of base stations.

PSS and SSS in LTE are sequences of 62 symbols transmitted over the central 72 resource elements of system bandwidth; hence synchronization signals occupy $1.08 \mathrm{MHz}$ of frequency resources by subcarrier spacing $15 \mathrm{kHz}$, independently of the channel bandwidth. Five consecutive resource elements are left unused at both ends of the occupied band while the direct current (DC) component is forced with null. The frequency allocation for PSS and SSS is showed in Figure 4. To detect PSS and SSS, the receiver needs 128 FFT points and minimum sample rate. The xSS signal structure is summarized in Table 3.

The location of synchronization signals is relative to both duplexing scheme and cycle prefix extension. In FDD, PSS is transmitted in the last OFDM symbol of slots 0 and slot 10 of the radio frames; and SSS is transmitted one OFDM symbol before the PSS allocation. The same PSS sequence is transmitted in the two slots; thus, the frame boundary cannot be detected without the half-frame uncertainty by using PSS only. But different PSS sequences are transmitted within one frame leading to the detection of the frame boundary. In addition, by detecting PSS and SSS it can be determined which duplexing technique is used. The reason is that the relative position between PSS and SSS is linked to the duplexing scheme. Thus, the knowledge of location of synchronization signals translates into the knowledge of duplexing scheme and it holds the other way around.

The mathematical model of the synchronization signals is found in [9].

\section{Physical broadcast channel}

The physical broadcast channel $(\mathrm{PBCH})$ is transmitted within the central six resource blocks. This allows the user equipment during the synchronization process to decode $\mathrm{PBCH}$ and read master information block (MIB) to ultimately get system bandwidth, which is needed to detect cell-specific reference signals.

Table 2. LTE-CRS signal parameters. Note: $\Delta_{p}=\Delta_{p}^{\prime} \Delta_{c}=90 \mathrm{kHz}$

\begin{tabular}{lllllllll}
\hline Channel BW $[\mathrm{MHz}]$ & $N_{p}$ & $\Delta_{p}^{\prime}$ & $N_{c}$ & $F_{s}[\mathrm{MHz}]$ & $T_{s}$ & $\beta$ & $\beta F_{s}[\mathrm{MHz}]$ & $T_{l}[\mathrm{~ms}]$ \\
\hline 1.4 & 12 & 6 & 128 & 1.92 & $0.52 \mu \mathrm{s}$ & 0.563 & 1.08 & 0.25 \\
3 & 30 & 6 & 256 & 3.84 & $0.26 \mu \mathrm{s}$ & 0.703 & 2.70 & 0.25 \\
5 & 25 & 6 & 512 & 7.68 & $0.13 \mu \mathrm{s}$ & 0.586 & 4.50 & 0.25 \\
10 & 100 & 6 & 1024 & 15.36 & $65.1 \mathrm{~ns}$ & 0.586 & 9.00 & 0.25 \\
15 & 150 & 6 & 1536 & 23.04 & $43.4 \mathrm{~ns}$ & 0.586 & 13.50 & 0.25 \\
20 & 200 & 6 & 2048 & 30.72 & $32.5 \mathrm{~ns}$ & 0.586 & 18.00 & 0.25 \\
\hline
\end{tabular}




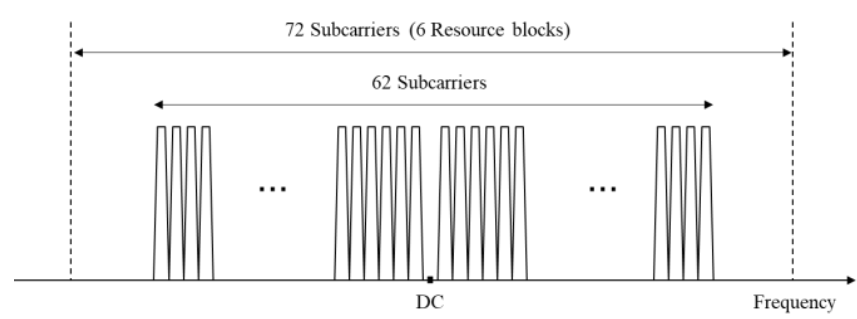

Figure 4. LTE PSS and SSS frequency allocation

Table 3. LTE PSS and SSS signal parameters. Note: $\Delta_{p}=\Delta_{p}^{\prime} \Delta_{c}=15 \mathrm{kHz}$

\begin{tabular}{lllllllll}
\hline Signal & $N_{p}$ & $\Delta_{p}^{\prime}$ & $N_{c}$ & $F_{S}$ & $T_{S}$ & $\beta$ & $\beta F_{S}$ & $T_{l}$ \\
\hline $\mathrm{xSS}$ & 62 & 1 & 128 & $1.92 \mathrm{MHz}$ & $0.52 \mu \mathrm{s}$ & 0.484 & $0.93 \mathrm{MHz}$ & $5 \mathrm{~ms}$ \\
\hline
\end{tabular}

\subsection{G signal structure}

The incoming 5G radio access technology aims at accommodating various equipment types and users, and extremely high data rate. Indeed, in addition to mobile terminal users, in this context, technologies such as autonomous vehicles and internet of things will also likely emerge and induce very specific traffic profile.

New radio 5G signals are designed to support a wide set of values of the subcarrier spacing (SCS). In this context, the term subcarrier spacing is often replaced with the term numerology. Then, we will use the two terms interchangeably in the rest of the paper. The numerology can be derived as

$$
\Delta_{f}^{\mu}=\Delta_{f} 2^{\mu}, \mu \in\{0,1, \ldots 4\}
$$

with $\mu$ the set of configuration indexes. The set of numerologies is summarized in Table 4, which shows how the subcarrier spacing increases as a function of the numerology index.

By using a larger subcarrier spacing the received signal becomes more robust against phase noise, therefore suitable for higher frequency transmissions and low-cost devices. Furthermore, increasing subcarrier spacing, since implies reducing symbol duration, suits low latency communication applications. Conversely, short symbol transmissions reduce robustness against multipath propagations as symbol duration relative to propagation delay decreases. This is not considered as an issue for higher frequency, since the propagation will likely be under line-of-sight conditions, due to the reduction of the cell size.

The normal cyclic prefix is supported by each numerology, but the extended cyclic prefix is supported only by numerology two. For the purpose of this paper we will consider only the normal cyclic prefix.

The radio frame structure that corresponds to the normal cycle prefix is shown in Figure 5. Here, frames and subframes have time duration constant, whereas slots and symbols have time duration that dependents on the numerology. Besides, the number of subframes within the frame and the number of symbols within the slots are constant, but the number of slots within the subframe is related to the numerology. This is summarized in Table 5. The physical layer resource grid is showed in Figure 6 for one subframe.

A resource grid is a composition of resource blocks which, in turn, is composition of resource elements. Each resource block consists of 12 consecutive subcarriers over the frequency domain and one OFDM symbol over the time domain. The frequency occupancy of one resource block increases as a function of the numerology. Inversely, the time occupancy of one resource block decreases as a function of the numerology. This is summarized in Table 6.

The number of configurable resource blocks, which defines the number of data subcarriers, and the total number of subcarriers, which corresponds to the number of the FFT points and which is directly linked to the system bandwidth, are both numerology dependent.

Table 4. Supported numerologies

\begin{tabular}{lll}
\hline $\begin{array}{l}\text { Numerology } \\
\text { index }(\mu)\end{array}$ & $\begin{array}{l}\text { Numerology } \\
{[\mathrm{kHz}]}\end{array}$ & $\begin{array}{l}\text { Cyclic } \\
\text { prefix }\end{array}$ \\
\hline 0 & 15 & $\mathrm{~N}$ \\
1 & 30 & $\mathrm{~N}$ \\
2 & 60 & $\mathrm{~N}, \mathrm{E}$ \\
3 & 120 & $\mathrm{~N}$ \\
4 & 240 & $\mathrm{~N}$ \\
\hline
\end{tabular}




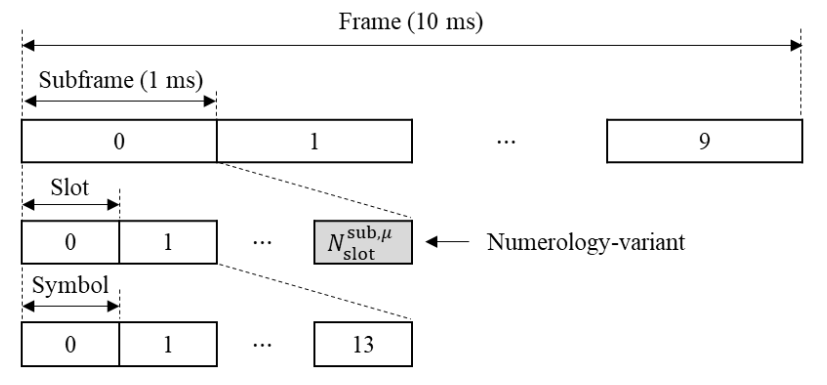

Figure 5. 5G frame structure

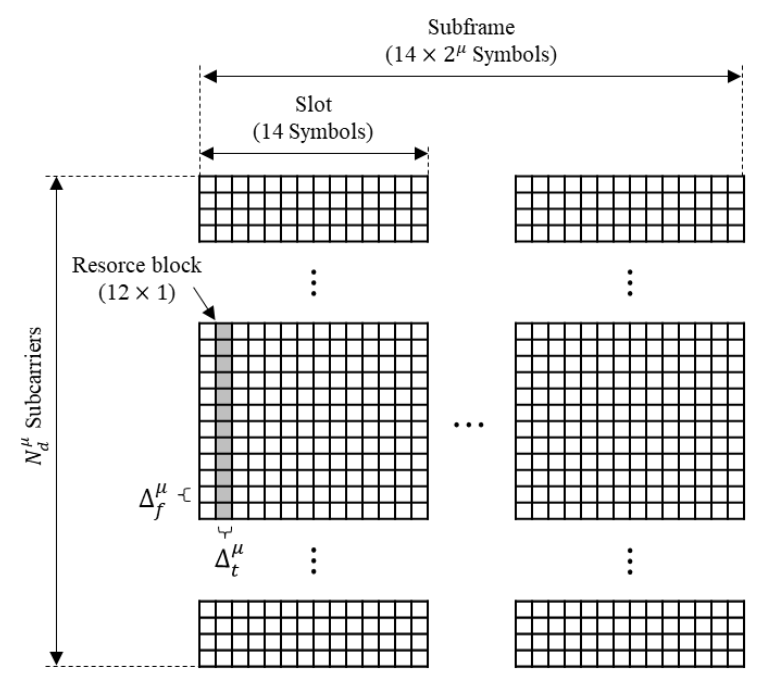

Figure 6. Physical layer resource grid

Table 5. Frame structure parameters [12]

\begin{tabular}{llll}
\hline$\mu$ & $\begin{array}{l}\text { Symbols / slot } \\
\left(N_{\text {sym }}^{\text {slt }}\right)\end{array}$ & $\begin{array}{l}\text { Slots / subframe } \\
\left(N_{\text {slot }}^{\text {sub } \mu}\right)\end{array}$ & $\begin{array}{l}\text { Slots / frame } \\
\left(N_{\text {slot }}^{\text {frm },}\right)\end{array}$ \\
\hline 0 & 14 & 1 & 10 \\
1 & 14 & 2 & 20 \\
2 & 14 & 4 & 4 \\
3 & 14 & 8 & 80 \\
4 & 14 & 16 & 160 \\
\hline
\end{tabular}

Table 6. Resource block dimension

\begin{tabular}{lllll}
\hline$\mu$ & $\begin{array}{l}\text { RB BW } \\
{[\mathrm{kHz}]}\end{array}$ & $\begin{array}{l}\text { RB durat. } \\
(\text { w/o CP) }[\mu \mathrm{s}]\end{array}$ & $\begin{array}{l}\text { CP durat. } \\
{[\mu \mathrm{s}]}\end{array}$ & $\begin{array}{l}\text { CP durat. } \\
(\text { sym. 0,7) }\end{array}$ \\
\hline 0 & 180 & 66.67 & 4.69 & NA \\
1 & 360 & 33.33 & 2.34 & NA \\
2 & 720 & 16.67 & 1.17 & NA \\
3 & 1440 & 8.33 & 0.57 & NA \\
4 & 2880 & 4.17 & NA & NA \\
\hline
\end{tabular}

\section{Frequency range}

In new radio, there are two separate frequency ranges respectively called FR1 and FR2. FR1 ranges from $410 \mathrm{MHz}$ to 7125 $\mathrm{MHz}$ and FR2 ranges from $24.25 \mathrm{GHz}$ to $52.6 \mathrm{GHz}$ [12], [13]. Each frequency range includes a certain number of operating bands. The frequency range FR2 is out of the scope of the paper, therefore we drop the description of the signal related to this frequency range. In the FR1, the higher carrier frequencies are placed within the operating bands 77, 78 and 79, which all are designed for commercial deployments.

\section{New radio system configuration}

In new radio, the total number of subcarriers, the number of data subcarriers and the sampling frequency can be configurated using the values in Table 7, 8 for respectively numerology 0,1 . The signals associated to numerology 2 are not covered in the context of this paper.

The sampling frequency which corresponds to the signal bandwidth can be derived as

$$
F_{s}^{\mu}=\Delta_{f}^{\mu} N_{c}^{\mu}, \mu \in\{0,1, \ldots 4\}
$$


Table 7. NR-FR1-N0 (SCS=15 kHz) signal parameters

\begin{tabular}{llll}
\hline $\begin{array}{l}\text { Channel } \\
\text { BW } \\
{[\mathrm{MHz}]}\end{array}$ & $\begin{array}{l}\text { Tot. } \\
\text { subcarriers } \\
\left(N_{c}^{\mu}\right)\end{array}$ & $\begin{array}{l}\text { Data } \\
\text { subcarriers } \\
\left(N_{d}^{\mu}\right)\end{array}$ & $\begin{array}{l}\text { Sampling } \\
\text { freq. }\left(F_{s}^{\mu}\right) \\
{[\mathrm{MHz}]}\end{array}$ \\
\hline 5 & 512 & 300 & 7.68 \\
10 & 1024 & 624 & 15.36 \\
15 & 1536 & 948 & 23.04 \\
20 & 2048 & 1272 & 30.72 \\
25 & 2048 & 1596 & 30.72 \\
30 & 3072 & 1920 & 46.08 \\
40 & 4096 & 2592 & 61.44 \\
50 & 4096 & 3240 & 61.44 \\
\hline
\end{tabular}

Table 8. NR-FR1-N1 (SCS=30 kHz) signal parameters

\begin{tabular}{llll}
\hline $\begin{array}{l}\text { Channel } \\
\text { BW } \\
{[\mathrm{MHz}]}\end{array}$ & $\begin{array}{l}\text { Tot. } \\
\text { subcarriers } \\
\left(N_{c}^{\mu}\right)\end{array}$ & $\begin{array}{l}\text { Data } \\
\text { subcarriers } \\
\left(N_{d}^{\mu}\right)\end{array}$ & $\begin{array}{l}\text { Sampling } \\
\text { freq. }\left(F_{s}^{\mu}\right) \\
{[\mathrm{MHz}]}\end{array}$ \\
\hline 5 & 256 & 132 & 7.68 \\
10 & 512 & 288 & 15.36 \\
15 & 768 & 456 & 23.04 \\
20 & 1024 & 612 & 30.72 \\
25 & 1024 & 780 & 30.72 \\
30 & 1536 & 936 & 46.08 \\
40 & 2048 & 1272 & 61.44 \\
50 & 2048 & 1596 & 61.44 \\
60 & 3072 & 1944 & 92.16 \\
70 & 3072 & $\mathrm{NA}$ & 92.16 \\
80 & 4096 & 2604 & 122.88 \\
90 & 4096 & 2940 & 122.88 \\
100 & 4096 & 3276 & 122.88 \\
\hline
\end{tabular}

\section{Synchronization signals and PBCH block}

To operate the initial cell search, the primary synchronization signal, PSS, and secondary synchronization signal, SSS, as well as the physical broadcast channel, $\mathrm{PBCH}$, are transmitted in the synchronization signal/PBCH (SS/PBCH) block or SS block (SSB) for simplicity.

The PSS and SSS consist of 127 subcarriers spanning from subcarrier number 56 through 182 and one OFDM symbol, respectively OFDM symbol number 0 and 2. Subcarriers number 0 through 47 and subcarrier number 192 through 239 in OFDM symbol number 2 are assigned to PBCH. The subcarriers between SSS and PBCH in OFDM symbol number 2 are left unused. OFDM symbols number 1 and 3 are assigned to $\mathrm{PBCH}$, which spans across 240 consecutive subcarriers from subcarrier number 0 through 239. The subcarrier and symbol indexes used in the description are defined in relation to the SSB start. The length of each component in an SSB is summarized in Table 9. The SSB structure is described in Figure 7. Here, the zerobased numbering used in the description to subcarriers and symbols is relative to the SSB start.

A network wise configurable number of SSBs is transmitted in the first half frame of the radio signal with periodicity of 2 frames [15], [16]. The frequency location of an SSB is assigned by the network based to the description in [16]. The SSB signal structure for FR1 is summarized in Table 10 . Note that the SSB is not supported by N2 [14].

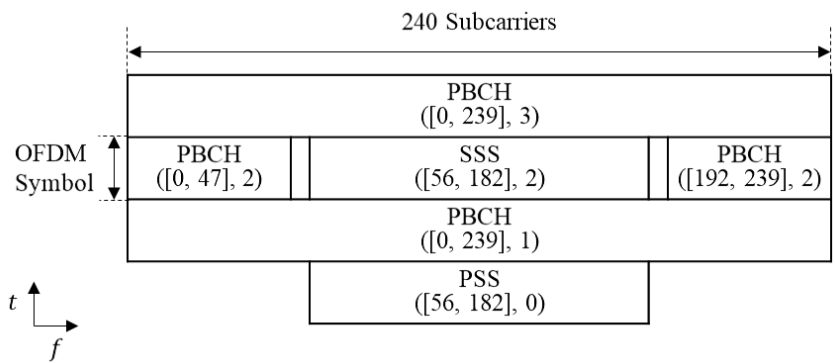

Figure 7. SSB structure. Adapted from primary source [14]

Table 9. SSB components

\begin{tabular}{lll}
\hline SSB component & Number of subcarriers & Symbol number \\
\hline PSS & 127 & 0 \\
SSS & 127 & 2 \\
PBCH & 240 & 1,3 \\
PBCH & 48,48 & 2 \\
Null & 8,9 & 2 \\
\hline
\end{tabular}


Table 10. SSB-FR1-N0 and SSB-FR1-N1 signal structure

\begin{tabular}{lllllllll}
\hline Signal & $N_{p}$ & $\Delta_{p}^{\prime}$ & $N_{c}$ & $F_{S}[\mathrm{MHz}]$ & $T_{S}[\mathrm{~ns}]$ & $\beta$ & $\beta F_{S}[\mathrm{MHz}]$ & $T_{l}[\mathrm{~ms}]$ \\
\hline $\mathrm{PSS}_{\mu=0}^{5 G}$ & 127 & $1(15 \mathrm{kHz})$ & 4096 & 61.44 & 16.27 & 0.031 & 1.90 & 20 \\
$\operatorname{PSS}_{\mu=1}^{5 G}$ & 127 & $1(30 \mathrm{kHz})$ & 4096 & 122.88 & 8.13 & 0.031 & 3.81 & 20 \\
$\operatorname{SSS}_{\mu=0}^{5 G}$ & 127 & $1(15 \mathrm{kHz})$ & 4096 & 61.44 & 16.27 & 0.031 & 1.90 & 20 \\
$\mathrm{SSS}_{\mu=1}^{5 G}$ & 127 & $1(30 \mathrm{kHz})$ & 4096 & 122.88 & 8.13 & 0.031 & 3.81 & 20 \\
$\mathrm{PBCH}_{\mu=0}^{5 G}$ & 240 & $1(15 \mathrm{kHz})$ & 4096 & 61.44 & 16.27 & 0.059 & 3.62 & 20 \\
$\mathrm{PBCH}_{\mu=1}^{5 G}$ & 240 & $1(30 \mathrm{kHz})$ & 4096 & 122.88 & 8.13 & 0.059 & 7.25 & 20 \\
\hline
\end{tabular}

\section{SIGNAL TRACKING PERFORMANCE}

In this section, the theoretical performance of signal tracking in a noisy single-path environment is evaluated and discussed.

\section{Simulation setup}

In this simulation, the one side equivalent loop filter bandwidth was set to $20 \mathrm{~Hz}$, the loop update spacing was set to the time separation between pilot symbols, as in Table 2, 3, 10, and the correlator spacing was set to 1 sample; the signal structure parameters $N_{p}, \beta$ and $F_{S}$ were set to as in Table 2, 3, 10; the SNR range, -20 to $35 \mathrm{~dB}$, includes values that even if below the minimum communication requirements still meet tracking.

The results were obtained using (18). They have been validated by real signals for LTE, not for 5G. The discussion of the results by real signals is out of the scope of the paper.

\section{Comparative results}

Figure 8 shows the standard deviation of the delay error estimate as a function of the signal to noise ratio for different mobile radio signals. The simulated signals are listed in Table 11. Here, LTE-CRS is for system bandwidth $20 \mathrm{MHz}$. The results show that the standard deviation of the delay estimate decreases logarithmically with the signal to noise ratio. At SNR $10 \mathrm{~dB}$, the standard deviation of the delay estimate for 5G-xSS-N0 and 5G-xSS-N1 is $3.68 \mathrm{~m}$ and $1.84 \mathrm{~m}$, respectively; for 5G-PBCH-N0 and 5G-PBCH-N1 is $1.41 \mathrm{~m}$ and $0.70 \mathrm{~m}$, respectively; for LTE-PSS and LTE-CRS is $5.44 \mathrm{~m}$ and $0.03 \mathrm{~m}$, respectively. This is summarized in Table 11, and synthetized as follows

- For paired numerologies, the standard deviation of delay error estimate for 5G-PBCH is 2.6 times smaller than the standard deviation of delay for $5 \mathrm{G}-\mathrm{xSS}$

- From N0 to N1 the standard deviation of the delay estimate is reduced by a factor of 2, due to the increase of the subcarrier spacing

- The minimum and maximum tracking performance in terms of systematic errors corresponds to LTE-xSS and LTECRS, respectively

- The maximum tracking performance for $5 \mathrm{G}$ corresponds to $5 \mathrm{G}-\mathrm{PBCH}-\mathrm{N} 1$

- The standard deviation for 5G-PBCH-N1 is 7.7 times smaller but 20.46 times larger than the standard deviation for LTE-xSS and LTE-CRS, respectively.

For reference signals that are spread all over the transmission bandwidth (LTE-CRS), the noise variance of the discriminator function decreases not only with the signal to noise ratio but also with the total number of subcarriers [1]. However, for reference signal that consists of constant number of pilot subcarriers (all other signals) the channel bandwidth does not influence the noise variance of the discriminator function.

Then, all 5G-RS within the same numerology and classification, regardless from which channel bandwidth configuration is used, provide the same systematic errors. The reason is that the ratio between the sampling frequency and total number of subcarriers, and the number of pilot subcarriers are both constants. Therefore, the performance indicator

$$
F_{s} \beta=F_{s} N_{p} \Delta_{p}^{\prime} / N_{c}=\Delta_{f} N_{p} \Delta_{p}^{\prime}
$$

is independent from the channel bandwidth. 


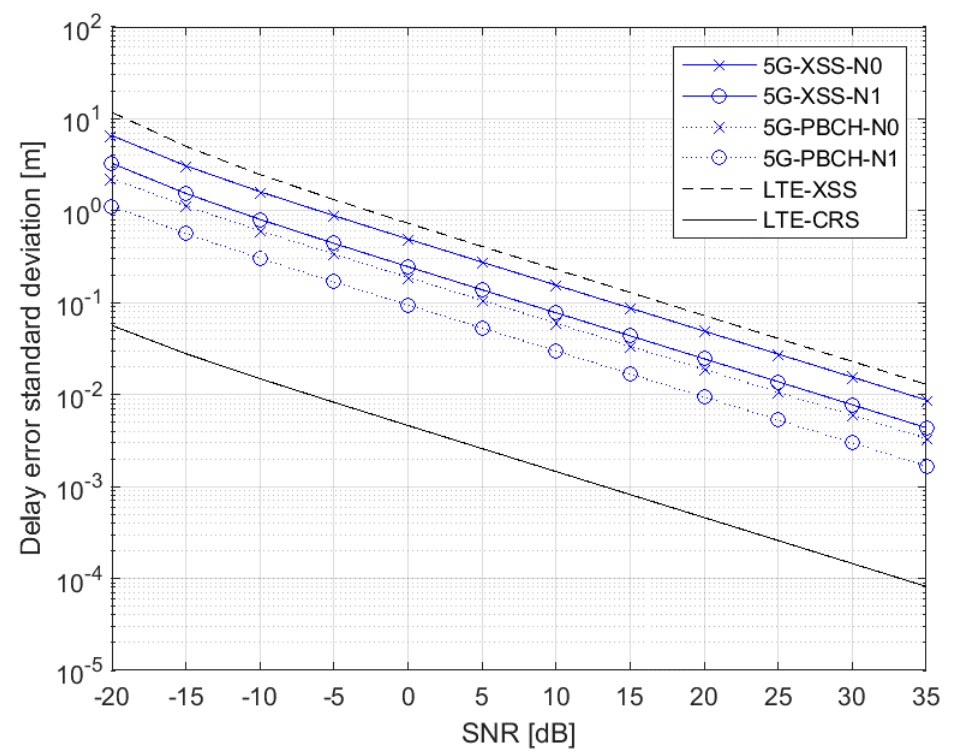

Figure 8. Description of systematic errors

Table 11. Standard deviation of delay error estimate $\left(\sigma_{\epsilon}\right)$ for different signals at SNR $10 \mathrm{~dB}$; gain factor $(G)$ relative to $5 \mathrm{G}$ PBCH-N1

\begin{tabular}{lll}
\hline Signal & $\sigma_{\epsilon}[\mathrm{cm}]$ & $G$ \\
\hline 5G-xSS-N0 & 15.5 & 5.216 \\
5G-xSS-N1 & 7.7 & 2.608 \\
5G-PBCH-N0 & 5.9 & 2 \\
5G-PBCH-N1 & 2.9 & 1 \\
LTE-xSS & 22.9 & 7.714 \\
LTE-CRS-20MHz & 0.1 & 0.049 \\
\hline
\end{tabular}

\section{CONCLUSION AND FUTURE WORK}

This paper demonstrated that 5G-microwave and LTE signals can be tracked using a common delay locked loop structure. The distinct features of the analyzed signals resulted in a different but comparable theoretical tracking performance. The standard deviation of the delay error estimate resulted small enough even at signal to noise ratio below the communication requirement, which means that the receiver does not necessarily need to be within the coverage area of the serving base station.

It is showed that using 5G-microwave signals the tracking performance get improved as the numerology get increased. It can be deduced using the results in this paper that higher numerology signals, namely 5G-millimeter signals, can be very much performing.

Among the considered signals, the maximum tracking performance for $5 \mathrm{G}$ regarded the $\mathrm{PBCH}$ signals. The validation of tracking based on PBCH signals needs to be addressed in the future work.

In this paper, it was assumed that in 5G there are no reference signals being spread all over the transmission bandwidth. In fact, this reduced the expected performance significantly.

Finally, the increase of the bandwidth due to the aggregation of multiple carrier components needs to be addressed in the future work.

\section{AKNOWLEDGMENT}

This work is a synergy between academia and industry. It has been supported financially by Orolia and the e-KnoT project, which are both gratefully acknowledged.

\section{REFERENCES}

[1] Shamaei, K. and Kassas, Z.M., "LTE Receiver Design and Multipath Analysis for Navigation in Urban Environments," Navigation, 65(4), 2018, pp.655-675. 
[2] Chen, L., Thevenon, P., Seco-Granados, G., Julien, O. and Kuusniemi, H., "Analysis on the TOA Tracking with DVBT Signals for Positioning," IEEE Transactions on Broadcasting, 62(4), 2016, pp.957-961.

[3] Serant, D., Thevenon, P., Boucheret, M.L., Julien, O., Macabiau, C., Corazza, S., Dervin, M. and Ries, L., "Development and Validation of an OFDM/DVB-T Sensor for Positioning," IEEE/ION Position, Location and Navigation Symposium, 2010, pp. 988-1001.

[4] Van de Beek, J.J., Sandell, M. and Borjesson, P.O., "ML Estimation of Time and Frequency Offset in OFDM systems," IEEE Transactions on Signal Processing, 45(7), 1997, pp.1800-1805.

[5] Cotter, S.F. and Rao, B.D, "Sparse Channel Estimation via Matching Pursuit with Application to Equalization," IEEE Transactions on Communications, 50(3), 2002, pp.374-377.

[6] Roy, R., Paulraj, A. and Kailath, T., "Estimation of Signal Parameters via Rotational Invariance Techniques-ESPRIT," MILCOM 1986-IEEE Military Communications Conference: Communications-Computers: Teamed for the 90's, vol. 3, 1986, pp. 41-6.

[7] Fleury, B.H., Tschudin, M., Heddergott, R., Dahlhaus, D. and Pedersen, K.I., "Channel Parameter Estimation in Mobile Radio Environments Using the SAGE Algorithm," IEEE Journal on Selected Areas in Communications, 1999, March, 17(3):434-450.

[8] Betz, J.W. and Kolodziejski, K.R, "Generalized Theory of Code Tracking with an Early-Late Discriminator Part I: Lower Bound and Coherent Processing," IEEE Transactions on Aerospace and Electronic Systems, 45(4), 2009, pp.1538-1556.

[9] 3GPP, "TS 36.211 V15.6.0, Release 15, Technical Specification Group Radio Access Network, Evolved Universal Terrestrial Radio Access (E-UTRA), Physical channels and modulation," 2019.

[10] ECO, "ECO Report 03: The Licensing of Mobile Bands in CEPT," 2018.

[11] 3GPP, "TS 36.101 V16.1.0, Release 16, Technical Specification, Evolved Universal Terrestrial Radio Access (EUTRA), User Equipment (UE) Radio Transmission and Reception," 2019.

[12] 3GPP, "TS 38.101-1 V15.5.0, Release 15, Technical Specification Group Radio Access Network, NR, User Equipment (UE) Radio Transmission and Reception; Part 1: Range 1 Standalone," 2019.

[13] 3GPP, "TS 38.101-2 V15.5.0; Release 15, Technical Specification Group Radio Access Network, NR, User Equipment (UE) radio transmission and reception, Part 2: Range 2 Standalone," 2019.

[14] 3GPP, "3GPP TS 38.300 V15.5.0, Release 15, Technical Specification Group Radio Access Network, NR and NG-RAN Overall Description," 2019.

[15] 3GPP, "3GPP TS 38.211 V15.5.0, Release 15, Technical Specification Group Radio Access Network, Physical channels and modulation," 2019.

[16] 3GPP, "3GPP TS 38.213 V15.5.0; Release 15, Technical Specification Group Radio Access Network, Physical layer procedures for control," 2019. 